\title{
Fluorescent Silica Nanoparticles with Well Separated Intensity Distributions from Batch Reactions
}

Teresa Kao ${ }^{\dagger}$, Ferdinand Kohle ${ }^{\dagger,}$, Kai Ma ${ }^{\dagger}$, Tangi Aubert ${ }^{\dagger,}$, , Alexander Andrievsky ${ }^{\dagger,}$,

Ulrich Wiesner ${ }^{\dagger}, *$

†Department of Materials Science and Engineering, Cornell University, Ithaca, NY 14853, USA

Department of Chemistry and Chemical Biology, Cornell University, Ithaca, NY 14853, USA

$\S$ Department of Chemistry, Ghent University, Ghent, 9000, Belgium

*Correspondence to Ulrich Wiesner. Email: ubw1@cornell.edu. Telephone: +1-607-255-3487.

Fax: +1-607-255-2365. 


\begin{abstract}
Silica chemistry provides pathways to uniquely tunable nanoparticle platforms for biological imaging. It has been a long-standing problem to synthesize fluorescent silica nanoparticles (SNPs) in batch reactions with high and low fluorescence intensity levels for reliable use as an intensity barcode, which would greatly increase the number of molecular species that could be tagged intracellularly and simultaneously observed in conventional fluorescence microscopy. Here, employing an amino-acid catalyzed growth, highly fluorescent SNP probes were synthesized with sizes $<40 \mathrm{~nm}$ and well separated intensity distributions, as mapped by single-particle imaging techniques. A seeded growth approach was used to minimize the rate of secondary particle formation. Organic fluorescent dye affinity for the SNP during shell growth was tuned using specifics of the organosilane linker chemistry. This work highlights design considerations in the development of fluorescent probes with well separated intensity distributions synthesized in batch reactions for single-particle imaging and sensing applications, where heterogeneities across the nanoparticle ensemble are critical factors in probe performance.
\end{abstract}

KEYWORDS: Fluorescent Probes, Silica Chemistry, Organic Fluorescent Dye, Total Internal Reflectance Fluorescence Microscopy, Single Particle Imaging, Multiplexed Imaging

Fluorescence microscopy has emerged as a powerful technique for non-invasive biological imaging ${ }^{1}$. Target molecules are typically visualized using fluorescent tags, such as fluorescent proteins $^{2,3}$, organic dyes, or nanoparticles ${ }^{4,5}$. Using spectrally distinct fluorescent tags, multiple molecular species can be labeled and simultaneously detected, facilitating studies of complex biological systems ${ }^{6}$. However, spectral multiplexing remains limited in many systems by the 
number of fluorescent tags that can be reliably distinguished using commercially available microscopes. This problem motivates a need for novel fluorescent tags that increase the number of molecular species that can be imaged in parallel, while maintaining the small sizes and target labeling capabilities of traditional fluorophores ${ }^{7}$.

Optical barcodes for highly multiplexed imaging have been realized in micron-sized beads exhibiting multiple spectral colors at distinguishable intensity levels ${ }^{8-10}$. These bead-based technologies typically rely on "bath dye" approaches for controlling fluorescence intensity, where solvent-swollen polymer particles are incubated in a solution of organic dye molecules, allowing dyes to absorb into the particles. Subsequent solvent removal traps dyes inside the particles ${ }^{9,10}$. Using micron-sized particles, intensity barcodes with up to ten distinguishable intensity levels can be generated simply by changing the concentration of dyes in the bath ${ }^{8-10}$. Despite wide success in micron-sized particles, the development of nano-sized intensity barcodes remains a challenge ${ }^{7}$. Highly-loaded microbeads may contain thousands of dyes per particle, allowing use of widely separated adjacent intensity levels ${ }^{8}$. For example, a factor of two difference in dye concentration may be used to generate microbeads that differ in dye-loading by an average of one thousand dyes. However, similar strategies cannot be applied in nano-sized systems, where small particle volumes necessitate use of substantially lower numbers of dyes per particle to prevent fluorescence quenching ${ }^{11}$. Under this constraint, a factor of two difference in dye concentration results in average differences of only a few dyes per particle and as illustrated in Figure 1, local fluctuations in dye concentration alone may cause overlap between intensity distributions ${ }^{10}$.

While previous reports have shown that this problem can be solved using programmable materials, including DNA and RNA ${ }^{7,12}$, only limited success in nanoparticle-based systems has been achieved ${ }^{13,14}$. Distinguishable intensity levels are difficult to achieve in nanoparticles using 
infiltration-based batch synthesis approaches even at increasing dye concentrations, due to low encapsulation efficiency ${ }^{15}$. Previous reports have shown that highly dye-doped nanoparticles with minimal fluorescence quenching may be synthesized by controlled covalent attachment of dyes during particle growth ${ }^{16}$. However, using these methods, the batch synthesis of nanoparticles with non-overlapping intensity distributions is complicated by heterogeneities across the ensemble, often exacerbated by secondary particle formation during growth ${ }^{17}$. Here, we have addressed these problems using a highly tunable synthesis method for covalently encapsulating organic fluorescent dyes inside silica nanoparticles (SNPs), by controlling secondary particle formation and varying the organosilane linker chemistry to the dye, demonstrating that high and low dye-loaded SNP intensity distributions are well separated as mapped by single particle fluorescence imaging.

Silica chemistry provides a uniquely tunable platform for nanoparticle synthesis, where particle size ${ }^{18-20}$, nanoscale morphology ${ }^{21}$, and surface properties ${ }^{22,23}$ can be precisely controlled. Recent advances in fabrication have extended the range of accessible particle sizes to below 10 $\mathrm{nm}$, using water as a solvent ${ }^{18}$, and established efficient methods for surface functionalization with a variety of targeting moieties ${ }^{22,23}$, making such materials ideal candidates as probes for biological imaging. We use a previously reported method for amino acid catalyzed growth of SNPs in aqueous media ${ }^{19,20}$ to investigate the synthetic parameters that govern single-particle intensity distributions in fluorescent dye-loaded SNPs. Using this basic framework, we demonstrate two different approaches for synthesizing bright SNPs with tunable average fluorescence intensity levels and sizes below $40 \mathrm{~nm}$. Controlled distributions of dyes within individual SNPs are achieved by incremental shell growth, allowing dyes to remain unquenched even at high loadings. The resulting SNPs are up to 100 times brighter than the constituent dyes. Optimized intensity distributions, as mapped by single-particle imaging techniques, are achieved by using a seeded 
growth approach to minimize the rate of secondary particle formation and by tuning the affinity of the organosilane linker for the SNP during shell growth to improve the incorporation efficiency of the often-expensive fluorescent dye molecules, by means of functional groups able to hydrogen bond to silica surface silanol groups.

Fluorescent SNPs were prepared either by homogenous or seeded growth in the presence of a silane-conjugated, fluorescent cyanine dye, Cy3 (Figure 2a) (see Supporting Information for detailed methods). We utilized l-arginine as a base catalyst for SNP formation in aqueous media at elevated temperatures of $60^{\circ} \mathrm{C}^{19,20,24}$. As previously studied in detail $1^{18,25}$, under such conditions (i.e. aqueous medium at elevated temperatures) silica nanoparticle formation proceeds via the rapid formation of small $(<2 \mathrm{~nm})$ silica clusters which subsequently aggregate into larger particles. In this system, however, a thin layer of tetraethoxysilane (TEOS) added to the top surface of the aqueous reaction volume serves as the silica source, with the interfacial area determining the rate of hydrolyzed TEOS diffusing into the water phase. For SNPs synthesized using what we call in the following the "homogenous" growth approach (Figures 2a and c), Cy3 dye was covalently coupled to an aminopropyltriethoxysilane (APTES) and added to the water phase before TEOS was layered on top. APTES was chosen for its relatively slow hydrolysis kinetics, as compared to aminopropyltrimethoxysilane (APTMS) ${ }^{26}$, to minimize self-aggregation and quenching of $\mathrm{Cy} 3$ dyes during particle nucleation. Due to the slow reaction rate of the sterically hindered Cy3/APTES conjugate, it is not fully consumed during the core synthesis. Three subsequent TEOS additions in $8 \mathrm{hr}$ increments were therefore used to allow for continued attachment of unreacted Cy3/APTES to SNPs, resulting in highly dye-doped particles. After growth was completed, SNPs were stabilized by covalent attachment of polyethylene glycol (PEG) chains ${ }^{22}$. SNPs were subsequently 
purified using gel permeation chromatography (GPC) to remove any unreacted chemicals ${ }^{18,23}$ (Figures S1c and d).

As a result of covalent dye encapsulation in the rigid silica matrix ${ }^{4,27,28}$, normalized absorption and emission spectra (Figures 3a) show that per dye brightness levels increase $\sim 7$ to 8 times over free dye in solution. Only slightly decreased per dye fluorescence enhancements are observed as the concentration of Cy3-silane is increased, suggesting that even at high loadings, dyes remain essentially unquenched. Cy3-labeled SNPs were further characterized using fluorescence correlation spectroscopy $(\mathrm{FCS})^{27}$, where fluctuations in fluorescence intensity generated by particle diffusion into and out of a detection volume of a confocal setup are autocorrelated and fit using a model describing single-component diffusion ${ }^{4,29,30}$. Importantly, a single FCS measurement provides information over hydrodynamic particle size, particle concentration, as well as particle brightness ${ }^{27}$, making it a powerful tool in fluorescent nanoparticle characterization. A $543 \mathrm{~nm}$ HeNe laser was used as the excitation source. Figure $3 \mathrm{~b}$ shows that the average hydrodynamic sizes obtained from FCS fits of Cy3-labeled SNPs synthesized by homogenous growth increase as Cy3/APTES concentration is increased. Using $1.3 \mu \mathrm{M}$ and 24.0 $\mu \mathrm{M}$ Cy3/APTES resulted in SNPs with diameters of $27.8 \mathrm{~nm}$ and $37.4 \mathrm{~nm}$, respectively.

The difference in particle size measured by FCS as a function of Cy3/APTES concentration is corroborated in the corresponding GPC elugrams (Figures S1c and d) of the particles. In this system, a typical GPC elugram exhibits three peaks as a function of elution volume, corresponding to particle aggregates, PEGylated particle product, and free reactants, respectively ${ }^{18}$. Shorter elution times were observed for SNPs prepared using $24.0 \mu \mathrm{M}$ Cy3/APTES (Figure S1d), indicating increased particle size relative to SNPs synthesized using 1.3 $\mu \mathrm{M}$ Cy3/APTES (Figure 
S1c). Transmission electron microscopy (TEM) images and corresponding size analysis (Figure S1a, b) corroborate the APTES-dependent size difference observed in FCS and GPC. Since the Cy3/APTES conjugate is prepared using a 10-fold excess of aminosilane and the reaction is performed at basic $\mathrm{pH}$, these observations are consistent with early aggregation of negatively charged silica clusters (vide supra) facilitated by increasing concentrations of positively charged APTES, resulting in increased average particle sizes ${ }^{25,31}$. Interestingly, the GPC elugram in Figure S1d for particles from the higher dye concentration (24.0 $\mu \mathrm{M}$ Cy3/APTES) exhibits a more skewed particle size peak. This suggests that higher concentrations of positively-charged APTES leads to continuous secondary particle formation, as more and more TEOS from the top layer hydrolyzes and diffuses into the water phase, thereby increasing particle size dispersity ${ }^{32}$. Despite increased polydispersity, Figure 3c clearly demonstrates that SNPs with increasing average fluorescence intensity levels could be obtained by adjusting the concentration of $\mathrm{Cy} 3$-silane used during particle synthesis, resulting in SNPs that are 15 and 47 times brighter than free Cy3 dye. Unfortunately, due to extreme particle size dispersity at even higher APTES levels, the concentration of Cy3silane could not be increased beyond $24.0 \mu \mathrm{M}$ without complete loss of particle uniformity.

Accurate identification of "high" and "low" intensity tags requires minimal overlap between single particle intensity distributions. We therefore evaluated batches of fluorescent dyeloaded SNPs for use as an intensity barcode, assessing single particle analysis based intensity distributions using total internal reflection fluorescence (TIRF) microscopy ${ }^{14}$. For imaging, biotinylated SNPs were immobilized on a streptavidin-coated glass substrate. Small numbers of biotin molecules were introduced to SNP surfaces during PEGylation using $\alpha, \omega-$ heterobifunctional PEG molecules (Figures 2a and c). Streptavidin-coated substrates were prepared using a previously established method $^{33}$ with slight modifications (see Supporting 
Information). Briefly, a thiol-coated substrate (blue in Figure 2b) was prepared by silanization of a glass slide (gray) using mercaptopropyltrimethoxysilane (MPTMS). N- $\gamma$-maleimidobutyryloxysuccinimide ester (GMBS) was attached to the thiol-coated substrate using its reactive maleimide group and the NHS ester group (green) at the opposite end of the GMBS was coupled to a primary amine on the streptavidin molecule (purple). Substrates were rinsed thoroughly after each step to remove unreacted chemicals. Imaging was performed over a 51.1 x $51.1 \mu \mathrm{m}^{2}$ area containing 100 SNPs. Single images were acquired for $100 \mathrm{msec}$. After image acquisition, a spot detection algorithm was used to identify individual SNPs. The background level and full width at half maximum (FWHM) of spots in the x- and y-directions were derived from Gaussian fits. Spot intensity was defined as the background-corrected sum over all pixels within the $\mathrm{FWHM}^{34}$ (see Supporting Information and Figures S2 and S3 for details).

We compared spot intensities from Cy3-labeled SNPs synthesized using the homogenous growth approach and $1.3 \mu \mathrm{M}$ or $24.0 \mu \mathrm{M}$ Cy3/APTES. The resulting histograms (Figure 4a), while showing an overall intensity increase for high Cy3-loaded particles, consistent with the increased average brightness/particle values measured using FCS (Figure 3c), substantially overlap. To benchmark the performance of these SNPs, we quantified the overlap between the two intensity distributions shown in Figure 4a. First, a threshold value was identified that minimizes overlap between high and low intensity distributions (dashed black line in Figure 4a). Overlap for high Cy3-loaded SNPs was then defined as the fraction of particles with spot intensity below that threshold value, while overlap for low Cy3-loaded SNPs was calculated as the fraction of particles with spot intensity above the threshold. Using these criteria, $15.3 \%$ and $13.4 \%$ overlap were calculated for high and low intensity distributions, respectively. This result indicates that on a single particle level, nearly $30 \%$ of particles cannot be unambiguously identified based on intensity 
alone. Clearly, such low identification accuracy would preclude use of these SNPs as an intensity barcode.

To overcome these limitations of the homogenous growth approach (i.e. high concentrations of Cy3/APTES causing extreme polydispersity in SNPs and limited separation from low particle brightness distributions), we introduced a second synthesis approach which we will refer to as the "seeded" growth method. Here, undyed SNPs with sizes $<20 \mathrm{~nm}$ were used as seeds for further particle growth (Figures $2 \mathrm{a}$ and d). Furthermore, Cy3 dye was covalently coupled to N-(2-aminoethyl)-3-aminopropyltrimethoxysilane (AEAPTMS) instead of APTES. The comparatively fast hydrolysis kinetics of AEAPTMS ${ }^{26}$ obviates the prolonged continued growth used to achieve high dye-loadings in the homogenous growth approach. Thus, to maintain relatively small particle sizes $(<40 \mathrm{~nm})$, only one additional TEOS growth step was used. SNPs were again PEGylated prior to purification using GPC. Seeded growth of SNPs was monitored using dynamic light scattering (DLS), demonstrating that incremental shell growth results in steadily increasing particle sizes (see Figure S4a in Supporting Information). Figure 3e shows that as compared to SNPs synthesized by homogenous growth (Figure 3b), the hydrodynamic sizes of SNPs synthesized by seeded growth do not vary significantly with increasing Cy3/AEAPTMS concentration. Using 6.0 $\mu \mathrm{M}, 24.0 \mu \mathrm{M}$, and 30.0 $\mu \mathrm{M}$ Cy3/AEAPTMS resulted in SNPs with diameters of $34.4 \mathrm{~nm}, 34.2 \mathrm{~nm}$, and $38.2 \mathrm{~nm}$, respectively. Using the same concentration of Cy3silane, SNPs prepared by seeded growth also exhibit more symmetric particle peaks in GPC (Figure S4e) than SNPs synthesized by homogenous growth (Figure S1d), suggesting that fewer secondary particles are formed. TEM images corroborated that these SNPs are narrowly dispersed (see Figure S4c and d in Supporting Information). 
Figure 3d shows that Cy3-labeled SNPs synthesized by seeded growth and using increasing amounts of Cy3/AEAPTMS exhibit per dye fluorescence enhancements of 6 to 7 times. As in SNPs synthesized via homogenous growth, increasing average brightness/particle was achieved by increasing the concentration of Cy3/AEAPTMS conjugate. Figure $3 \mathrm{f}$ shows that Cy3-labeled SNPs using $6.0 \mu \mathrm{M}, 24.0 \mu \mathrm{M}$, and $30.0 \mu \mathrm{M}$ Cy3/AEAPTMS are 28, 91, and 106 times brighter, respectively, than free Cy3 dye. Comparison between Figures $3 \mathrm{c}$ and f shows that from $24.0 \mu \mathrm{M}$ Cy3-silane concentration upwards, the seeded growth approach is a more efficient method of generating bright fluorescent tags. Cy3-labeled SNPs prepared by seeded growth using $24.0 \mu \mathrm{M}$ Cy3/AEAPTMS are nearly 2 times brighter than those synthesized by homogenous growth using Cy3/APTES at the same concentration, even though the per dye brightness is slightly decreased in these particles. This increased dye incorporation efficiency is attributed to reduced rates of secondary particle nucleation/formation achieved using the seeded growth approach and to the relatively fast reaction rates of $\mathrm{Cy} 3 / \mathrm{AEAPTMS}$ as compared to Cy3/APTES. As this result could not be achieved with Cy3/APTMS (data not shown), we hypothesize that the secondary amines present in AEAPTMS may also contribute to the enhanced association with the growing SNPs by hydrogen bonding to silica surface silanol groups ${ }^{35}$, further increasing the incorporation efficiency of Cy3/AEAPTMS relative to Cy3/APTES.

The advantages of the seeded growth of fluorescent SNPs using Cy3/AEAPTMS (i.e. increased brightness/particle in FCS) particularly manifested in TIRF microscopy. Single particle intensity distributions for SNP batches synthesized using $6.0 \mu \mathrm{M}, 24.0 \mu \mathrm{M}$, and $30.0 \mu \mathrm{M}$ Cy3/AEAPTMS show that an overall intensity increase is reproducibly observed as the concentration of Cy3/AEAPTMS is increased (see Figure S5 in the Supporting Information). Importantly, single particle intensity distributions for batches generated using the seeded growth 
approach and $24.0 \mu \mathrm{M} \mathrm{Cy3/AEAPTMS} \mathrm{(Figure} \mathrm{S5b)} \mathrm{are} \mathrm{also} \mathrm{shifted} \mathrm{to} \mathrm{higher} \mathrm{spot} \mathrm{intensities} \mathrm{as}$ compared to particle batches derived from the homogeneous growth approach at the same concentration of Cy3/APTES (Figure 4a). Designating SNP batches synthesized by homogenous (1.3 $\mu \mathrm{M}$ Cy3/APTES) and seeded growth, (30.0 $\mu \mathrm{M}$ Cy3/AEAPTMS) as low and high intensity level tags, we approach near perfect identification accuracy. Figure $4 \mathrm{~d}$ shows that single particle intensity distributions exhibit 0.2 to $0.3 \%$ overlap for low (purple) and high (green) Cy3-loaded SNPs, indicating that $>99 \%$ of SNPs are unambiguously identifiable based on intensity alone. We applied our intensity barcoding scheme to fluorescence images of mixed high and low intensity probes (Figure 4c). Spots above and below the intensity threshold defined in Figure 4d are colored green and purple, respectively. Comparison to images of only high (Figure 4e) or only low intensity (Figure 4b) probes illustrates that highly accurate intensity-based identification can be achieved. While for these proof-of-principle demonstrations, batches from homogeneous and seeded growth were compared, we expect that after careful parameter optimization other combinations, for example of two batches synthesized using seeded growth, will show similar results.

We have described two batch approaches for the synthesis of fluorescent silica nanoparticles using either homogenous or seeded growth. Controlled fluorescence intensity levels can be achieved in these systems simply by varying the concentration of a Cy3-silane conjugate in the batch. We have shown that the precise spatial distributions of dyes within particles can be controlled by silica shell growth, minimizing self-quenching of dyes even in highly dye-doped particles. Comparison of the two approaches highlights that the nucleation of secondary particles during shell growth presents a major challenge in the synthesis of highly dye-doped SNPs with narrowly distributed single particle fluorescence intensity distributions. Using the seeded growth 
approach, we have decreased the rate of secondary nucleation. Simultaneously, we have increased the rate of $\mathrm{Cy} 3$ incorporation by choice of silane linker (e.g. AEAPTMS versus APTES), minimizing the effects of secondary nucleation in this system.

We find that single particle analysis based fluorescence intensity distributions of SNPs synthesized in these ways are well separated and can therefore be used as an intensity-based barcode. These SNPs are small $(<40 \mathrm{~nm})$, highly biocompatible, and readily functionalizable, making them ideal fluorescent tags for imaging in living biological systems. We have used a single fluorescent dye (Cy3) as proof-of-concept, but expect that our results can be extended to combinations of dyes with multiple spectral colors, greatly increasing the number of SNP tags that can be imaged in parallel using conventional fluorescence microscopy. Additionally, since this strategy relies on the improved incorporation efficiency of a silane linker, we expect that similar results can be achieved using a range of organic fluorescent dyes and linker chemistries, providing an efficient route to encapsulation of these often-expensive molecules. 
(a)
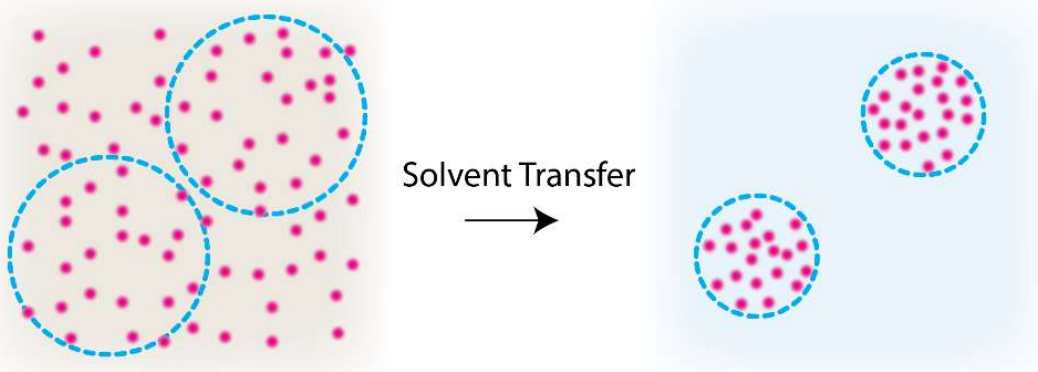

(b)
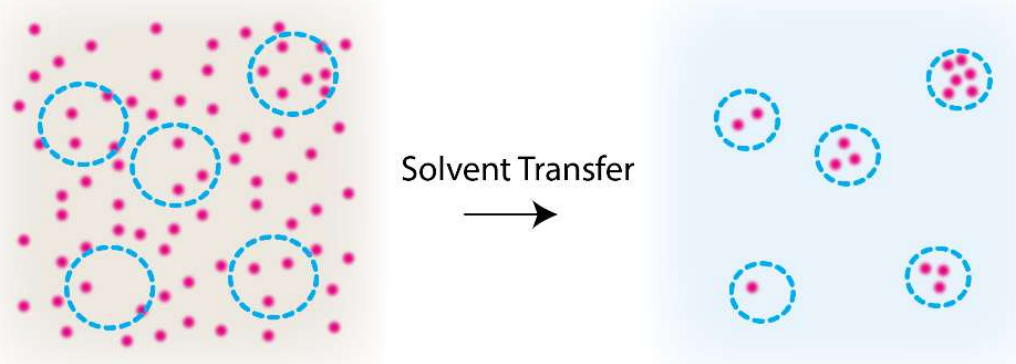

Figure 1. Schematic illustration of a bath dye approach for synthesizing fluorescent polymer beads. $(a, b)$ Solvent-swollen polymer particles (blue circles) are incubated in a solution of organic dye molecules, allowing dyes to absorb into particles. Solvent transfer traps dyes inside the particles. Comparison of large (a) and small (b) particles shows that decreasing the average number of encapsulated dyes in small particles magnifies the effects of local concentration fluctuations on the number of dyes per particle leading to large heterogeneities. 
(a)

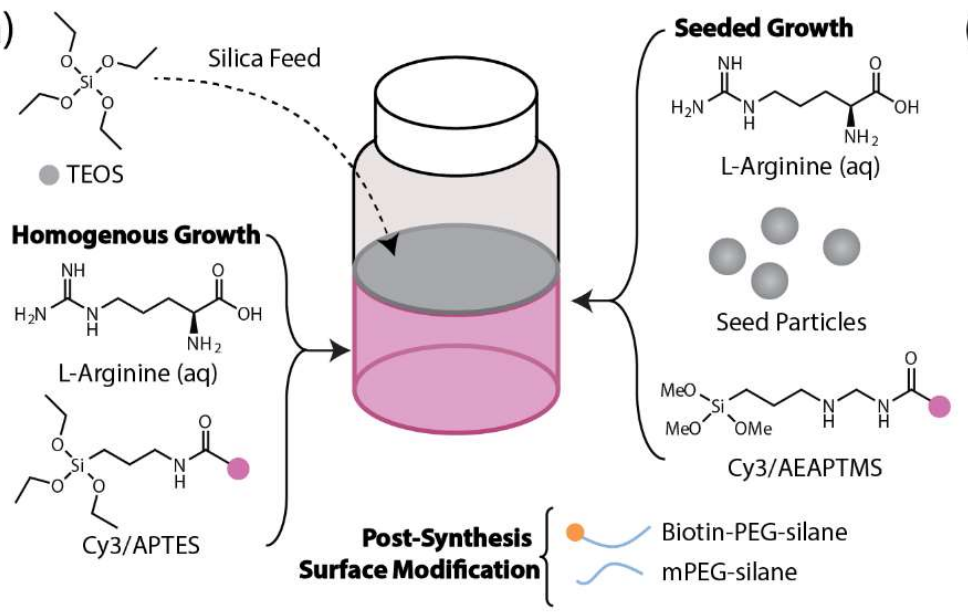

(b)

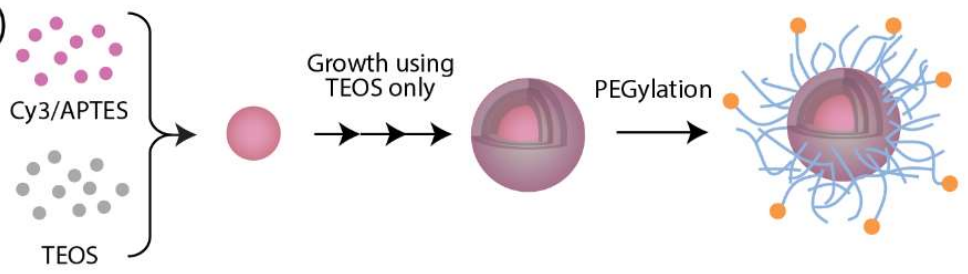

(c)

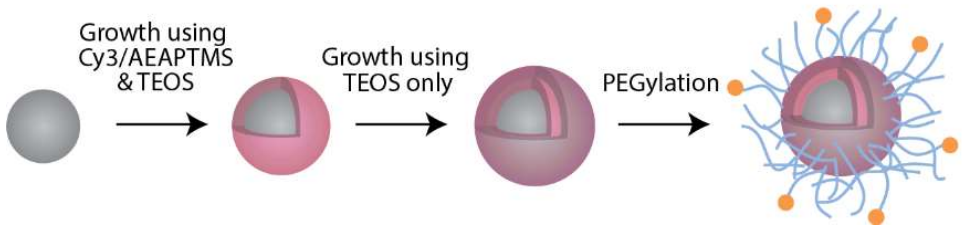

(d)

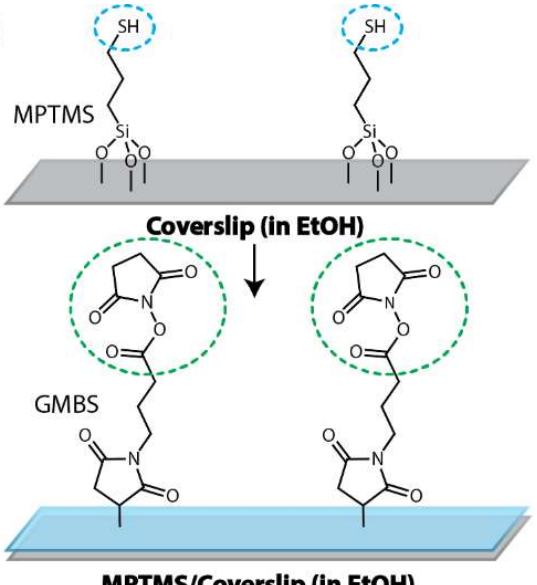

MPTMS/Coverslip (in EtOH)

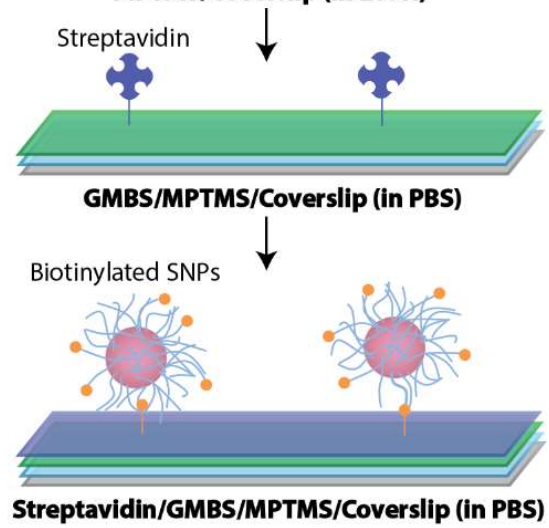

Figure 2. Schematic representations of the preparation of fluorescent SNPs and substrates for TIRF microscopy. (a) Experimental setup and chemicals used during synthesis. (b, c) Growth pathways of fluorescent SNPs synthesized using (b) homogenous and (c) seeded growth. (d) For single particle imaging, a thiol-coated substrate (blue) was prepared by incubating a glass slide (gray) in a solution of MPTMS in ethanol (EtOH). Heterobifunctional GMBS was attached to the thiol-coated substrate using its reactive maleimide group and the NHS ester group (green) at the opposite end was coupled to a primary amine on the streptavidin molecule (purple). Substrates were rinsed thoroughly after each step to remove unreacted chemicals. Biotinylated SNPs were attached to streptavidin-coated substrates just before imaging. 

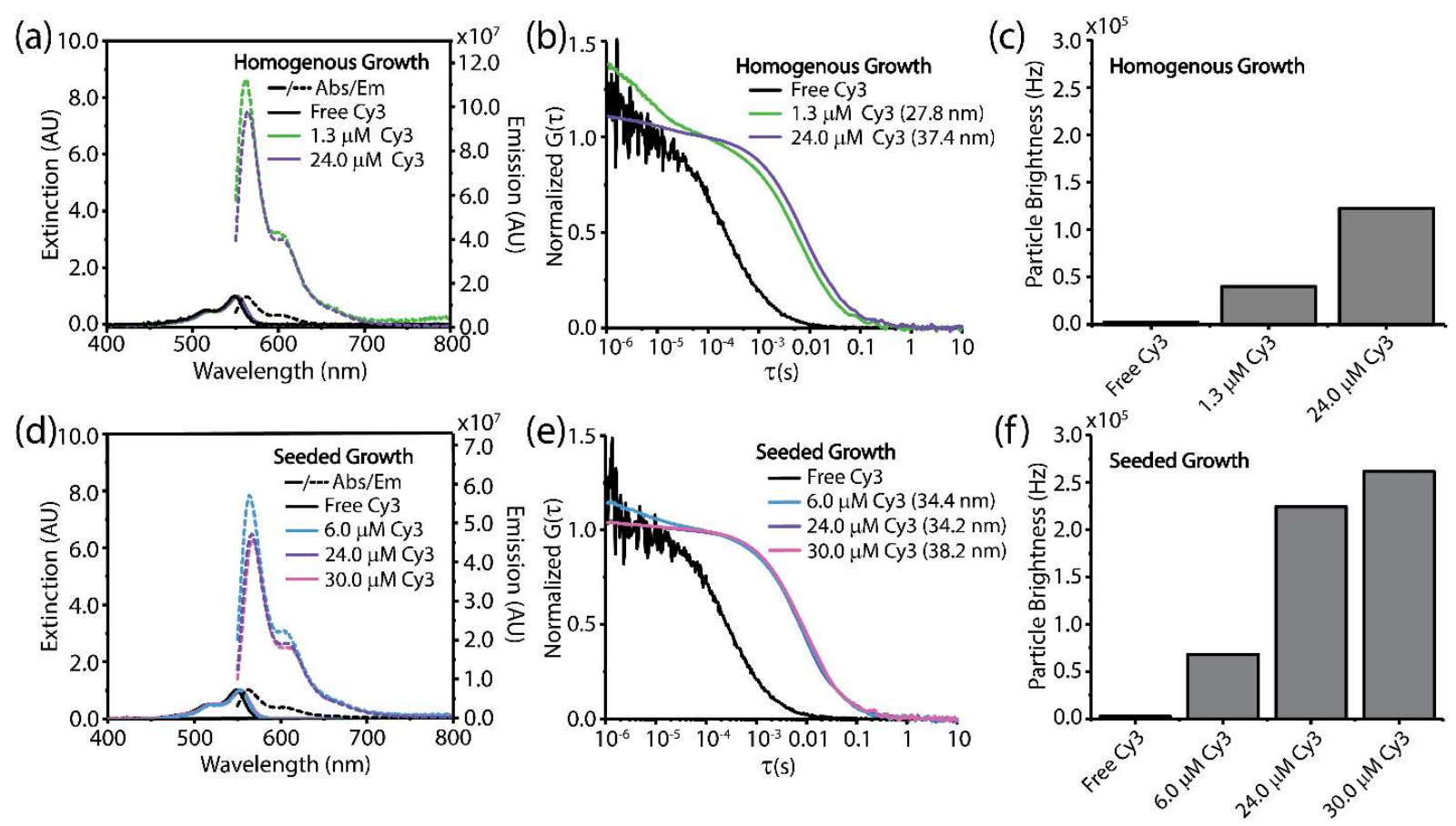

Figure 3. Characterization of fluorescent SNPs synthesized by (a-c) homogenous and (d-f) seeded growth. (a, d) Absorbance (solid lines) and emission spectra (dashed lines), (b, e) FCS curves, and (c, f) FCS-derived per particle brightness values for SNPs synthesized using homogenous or seeded growth and varying Cy3/APTES or Cy3/AEAPTMS concentrations, respectively. The emission spectra in (a) and (d) are truncated on the left side because overlap with the $543 \mathrm{~nm}$ excitation source prevents direct quantification of the sample emission at these wavelengths. Noise levels of FCS curves for free dye in (b) and (e) are higher than for particles due to substantially increased particle brightness, see (a) and (d). 

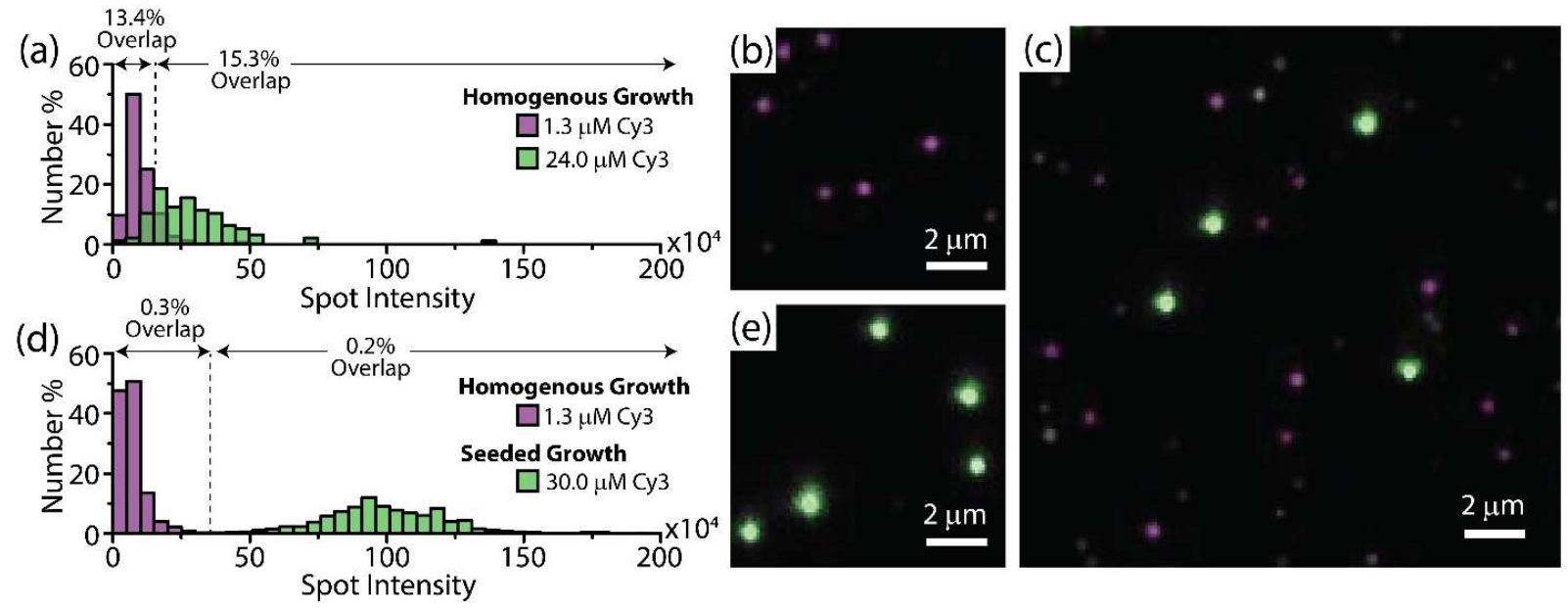

Figure 4. Comparison of fluorescence intensity levels for low and high dye-loaded SNPs as revealed by single particle imaging using TIRF microscopy. (a, d) Single-particle intensity distributions for SNPs. The SNPs shown in (a) are prepared by homogenous growth, using $1.3 \mu \mathrm{M}$ (purple) and $24.0 \mu \mathrm{M}$ (green) Cy3/APTES. Overlap for high Cy3-loaded SNPs is defined as the fraction of particles with spot intensity below a threshold value (dashed black lines). Overlap for low Cy3-loaded SNPs is calculated as the fraction of particles with spot intensity above the same threshold value. In (d), low Cy3-loaded SNPs are again prepared by homogenous growth, using $1.3 \mu \mathrm{M}$ (purple) Cy3/APTES. High Cy3-loaded SNPs are synthesized using seeded growth and $30.0 \mu \mathrm{M}$ Cy3/AEAPTMS (green). (b, c, e) Single particle fluorescence images of (b) only the low, (e) only the high, and (c) mixtures of the low and high Cy3-loaded SNPs shown in (d). Spots above and below the threshold shown in (d) are colored green and purple, respectively. 


\section{ASSOCIATED CONTENT}

\section{Supporting Information}

The Supporting Information is available free of charge on the ACS Publications website. Materials, synthetic methods, characterization, and image analysis are described. Additional characterization of SNPs prepared by homogenous and seeded growth is shown. Datasets showing reproducibility of the synthesis and imaging method are also included.

\section{AUTHOR INFORMATION}

\section{Corresponding Author}

*E-mail: $\underline{\text { ubw1@cornell.edu }}$

\section{Funding Sources}

Research reported in this publication was supported by the National Cancer Institute of the National Institutes of Health under Award Number U54CA199081. The authors gratefully acknowledge Dr. H. Ow for initial input and discussions on the synthesis method used in this paper. F.K. acknowledges financial support by the U.S. Department of Energy, Office of Science, Basic Energy Sciences, under Award No. DESC0010560. T.A. acknowledges financial support by the Ghent University Special Research Fund (BOF14/PDO/007) and the European Union's Horizon 2020 research and innovation program (MSCA-IF-2015-702300). This work made use of the CCMR Shared Facilities, which are supported through the NSF MRSEC program (DMR1120296). This work made use of the Nanobiotechnology Center shared research facilities at Cornell. Imaging data was acquired through the Cornell University Biotechnology Resource Center, with NSF funding (1428922) for the shared Zeiss Elyra Microscope. 


\section{REFERENCES}

1. Lichtman, J. W.; Conchello, J. Nat. Meth. 2005, 2, 910 - 919.

2. Joo, C.; Balci, H.; Ishitsuka, Y.; Buranachai, C.; Ha, T. Annu. Rev. Biochem. 2008, 77, 51 $-77$.

3. Tsien, R. Y. Annu. Rev. Biochem. 1998, 67, $509-544$.

4. Ow, H.; Larson, D.; Srivastava, M.; Baird, B., Webb, W.; Wiesner U. Nano Lett. 2005, 5, $113-117$.

5. Medintz, I. L.; Uyeda, H. T.; Goldman, E. R.; Mattoussi, H. Nat. Mater. 2005, 4, 435 446.

6. Lubeck, E.; Cai, L. Nat. Methods. 2012, 9, $743-748$.

7. Woehrstein, J. B.; Strauss, M. T.; Ong, L. L.; Wei, B.; Zhang, D. Y.; Jungmann R.; Yin P. Sci. Adv. 2017, 3, 1602128.

8. Han, M.; Gao, X.; Su, J. Z.; Nie, S. Nat. Biotechnol. 2001, 19, $631-635$.

9. Zhang, Y. Z.; Kemper, C. R.; Haugland, R.P. Microspheres with fluorescent spherical zones. US 5,786,219 (1998).

10. Banerjee, S.; Georgescu, C.; Daniels, E. S.; Dimonie, V. L., Seul, M. Production of dyed polymer microparticles. US 6,964,747 (2005).

11. Forster, T.; Ann. Phys. 1948, 437, 55 - 75.

12. Um, S. H.; Lee, J. B.; Kwon, S. Y.; Li, Y.; Luo D. Nat. Protoc. 2006, 1, $995-1000$.

13. Wang, L.; Tan, W. Nano Lett. 2006, 6, $84-88$.

14. Wu, C.; Hansen, S. J.; Hou, Q.; Yu, J.; Zeigler, M.; Jin, Y.; Burnham, D. R.; McNeill, J. D.; Olson, J. M.; Chiu, D. T. Angew. Chem. Int. Ed. 2011, 50, 3430 - 3434.

15. Jungmann, N.; Schmidt, M.; Ebenhoch, J.; Weis, J.; Maskos, M. Angew. Chem., Int. Ed. 2003, 42,1714

16. Ow, H.; Larson, D.; Srivastava, M.; Baird, B., Webb, W.; Wiesner U. Nano Lett. 2005, 5, $113-117$.

17. Graf, C.; Vossen, D. L. J.; Imhof, A.; van Blaaderen, A. Langmuir. 2003, 19, 6693 6700 .

18. Ma, K.; Mendoza, C.; Werner-Zwanziger, U.; Zwanziger, J.; Wiesner, U. Chem. Mater. 2015, 27, $4119-4133$. 
19. Yokoi, T.; Sakamoto, Y.; Terasaki, O.; Kubota, Y.; Okubo, T.; Tatsumi, T. J. Am. Chem. Soc. 2006, $128,13644-13665$.

20. Hartlen, K. D.; Athanasopoulos, A. P. T.; Kitaev, V. Langmuir. 2008, 24, 1714-1720.

21. Ma, K.; Sai, H.; Wiesner, U. J. Am. Chem. Soc. 2012, 134, 13180 - 13183.

22. Ma, K.; Zhang, D.; Cong, Y.; Wiesner, U. Chem. Mater. 2016, 28, 1537 - 1545.

23. Ma, K.; Wiesner, U. Chem. Mater. 2017, 29, 6840 - 6855.

24. Schubbe, S.; Cavelius, C.; Schumann, C.; Koch, M.; Kraegeloh, A. Adv. Eng. Mater. 2010, $12,417-422$.

25. Carcouët, C. C. M. C.; van de Put, M. W. P.; Mezari, B.; Magusin, P. C. M. M.; Laven, J.; Bomans, P. H. H.; Friedrich, H.; Catarina, A.; Esteves, C.; Sommerdijk, N. A. J. M.; van Benthem, R. A. T. M.; de With, G. Nano Lett. 2014, 14, 1433 - 1438.

26. Wang, J.; Sugawara-Narutaki, A.; Fukao, M.; Yokoi, T.; Shimojima, A.; Okubo, T. ACS Appl. Mater. Interfaces, 2011, 3, 1538 - 1544.

27. Larson, D. R.; Ow, H.; Vishwasrao, H. D.; Heikal, A. A.; Wiesner, U.; Webb, W. W. Chem. Mater. 2008, 20, 2677 - 2684.

28. Muddana, H. S.; Morgan, T. T.; Adair, J. H.; Butler, P. J. Nano Lett. 2009, 9, $1559-$ 1566.

29. Magde, D.; Elson, E.; Webb, W. W. Phys. Rev. Lett. 1972, 29, 705.

30. Yin, Y.; Yuan, R.; Zhao, X. S. J. Phys. Chem. Lett. 2013, 4, $304-309$.

31. Mizutani, T.; Nagase, H.; Fujiwara, N.; Ogoshi, H. Bull. Chem. Soc. Jpn. 1998, 71, 2017 $-2022$.

32. Chen, C; Hayakawa, C; Shirosaki, Y.; Fujii, E.; Kawabata, K; Tsuru, K; Osaka, A. J. Am. Ceram. Soc. 2009, 92, $2074-2082$.

33. Ngundi, M. M.; Shriver-Lake, L. C.; Moore, M. H.; Lassman, M. E.; Ligler, F. S.; Taitt, C. R. Anal. Chem. 2005, 77, $148-154$.

34. Willing, K. I.; Rizzoli, S. O.; Westphal, V.; Jahn, R.; Hell, S.W. Nature. 2006, 440, 935 939.

35. Kroger, N.; Deutzmann, R.; Bergsdorf, C.; Sumper, M. Proc. Natl. Acad. Sci. 2000, 97, $14133-14138$. 